Abstract The high field strength of femtosecond laser pulses leads to nonlinear effects during the interaction with condensed matter. One such effect is the ablation process, which can be initiated below the threshold of common thermal ablation if the excitation pulses are sufficiently short. This effect leads to structure formation, which is anisotropic because of the polarization properties of the near field and can result in pattern sizes below the resolution limit of light. These effects are explored by temporally resolved scattering methods and by post-mortem analysis to show the non-thermal and anisotropic nature of this process The near-field distribution of plasmon modes can be tailored to a large extent in order to obtain control of the pattern formation.

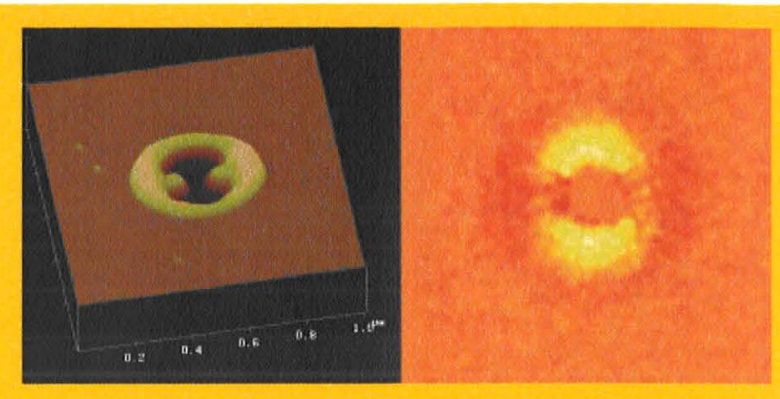

Ablation impact of a spherical polystyrene particle on a silicon surface (left) as visualized by atomic force microscopy and the change in the scattering pattern of a gold nanoparticle (right) both irradiated with single femtosecond pulses. The anisotropy in both images is a result of the near-field-enhanced sub-threshold ablation process.

\title{
Femtosecond laser near field ablation
}

\author{
Anton Plech ${ }^{1,2, *}$, Paul Leiderer ${ }^{2}$ and Johannes Boneberg ${ }^{2}$ \\ ${ }^{1}$ Institut für Synchrotronstrahlung (ISS), Karlsruhe Institute of Technology, Postfach 3640, 76021 Karlsruhe, Germany \\ ${ }^{2}$ Center for Applied Photonics, Universität Konstanz, Universitätsstr. 10, 78457 Konstanz, Germany
}

Key words: Nanostructures, ablation, femtosecond laser, plasmon, near-field enhancement, X-ray scattering, sub-wavelength structures.

PACS: $52.38 . \mathrm{Mf}, 61.05 . \mathrm{cf}, 73.20 . \mathrm{Mf}$

\section{Introduction}

Photonic applications with nanostructures have seen a large increase in interest within the last years. A wealth of phenomena is connected to the linear interactions of light with structural features of materials below the wavelength of light. Photonic crystals are such key elements that hold the promise to manipulate light and quantum objects in a similar manner as in semiconductor electronics. Plasmonics is a second field that targets the enhancement effects of light and the boost of nonlinear reactions compared to the linear description. Ultra-short lasers are key components in this field as they favor field-dependent reactions relative to intensity-dependent pathways. One pathway for material machining is the ablation process. In general, laser ablation by pulsed sources is considered as a non-equilibrium thermal phenomenon, whereby the rapid deposition of heat into the material leads to melting and boiling.

The energy is deposited in the material fast enough so that energy dissipation can be neglected. In this case the material is isochorically superheated to temperatures above the boiling point. Phase separation sets in during the expansion phase, when the system crosses the liquid-gas coexistence. As a consequence, material is ejected violently due to the increased vapor pressure. Transparent materials can be structured by ablation if the laser fluence exceeds the plasma-formation threshold. Multiphoton effects play a prominent role in the energy deposition. Conceptually new phenomena can arise if the structures are smaller than the wavelength of light and the electromagnetic energy is concentrated by so-called near-field effects. While in the far field the concentration of energy is limited by the wavelength of the radiation, on a local scale a large en-

* Corresponding author: e-mail: anton.plech@uni-konstanz.de 
hancement can occur at curved interfaces to a spatial scale much smaller than the wavelength.

This article reviews work done to establish the nearfield enhanced ablation on nanostructures and structured surfaces. A particular emphasis is given on the determination of the fundamental mechanism, which includes the definition of thermal and non-thermal phenomena. The post-mortem analysis of ablated patterns from structured materials can serve as an imaging method for near-field distributions, while an unique time resolved X-ray scattering technique has been employed to follow the structural dynamics with high time resolution.

Sect. 2 will serve to summarize the basic mechanism in the general laser ablation process, in particular with short pulses. In Sects. 3 and 4 the interaction of highly curved structures with electromagnetic waves will be sketched, which leads to the definition of the field enhancement and plasmon resonances. Sect. 5 will demonstrate that the structuring mechanism on nanoparticles can be understood as a nonlinear process with strong correlations to an ablation process, but with a non-thermal mechanism behind it. The relevance of using particles on surfaces for structuring issues by taking advantage of the nonlinearity is finally presented in Sect. 6.

\section{Recapitulation of thermal ablation from surfaces}

Laser-based ablation processes are of eminent importance for contactless machining of materials and are therefore well studied [1-3]. In particular, short-pulse ablation with picosecond or femtosecond pulses (nanosecond pulses in the case of soft matter) can yield very selective and localized material emission due to the confinement conditions at early times of the excitation. Thus, the heataffected area near laser-drilled holes can be minimized, or organic materials can be ablated very gently for use in molecular diagnostics (such as matrix-assisted laser desorption/ionization) [4].

Opaque materials can be easily heated to temperatures above the boiling point of the material by short laser pulses. The heating of surfaces is isochoric, i.e. under volume conservation for laser pulse lengths shorter than the acoustic transient time of the excited material. If during the heating time the temperature of the material exceeds the boiling temperature a violent expansion and nucleation of vapor bubbles will occur. This phase change, often called explosive boiling or phase explosion, supplies the pressure for the ejection of the heterogeneous material. It can be shown by experiments or molecular dynamics studies [5-8] that this nucleation causes the emission of material clusters before sublimation starts to set in [9]. In particular, simulations are extremely helpful to visualize the dynamies during thermal ablation, as shown in Fig. 1. An application of this effect is the production of nanoparticles from irradiating solid targets of a large variety of materials, which can be done in vacuum as well as in liquids [10]
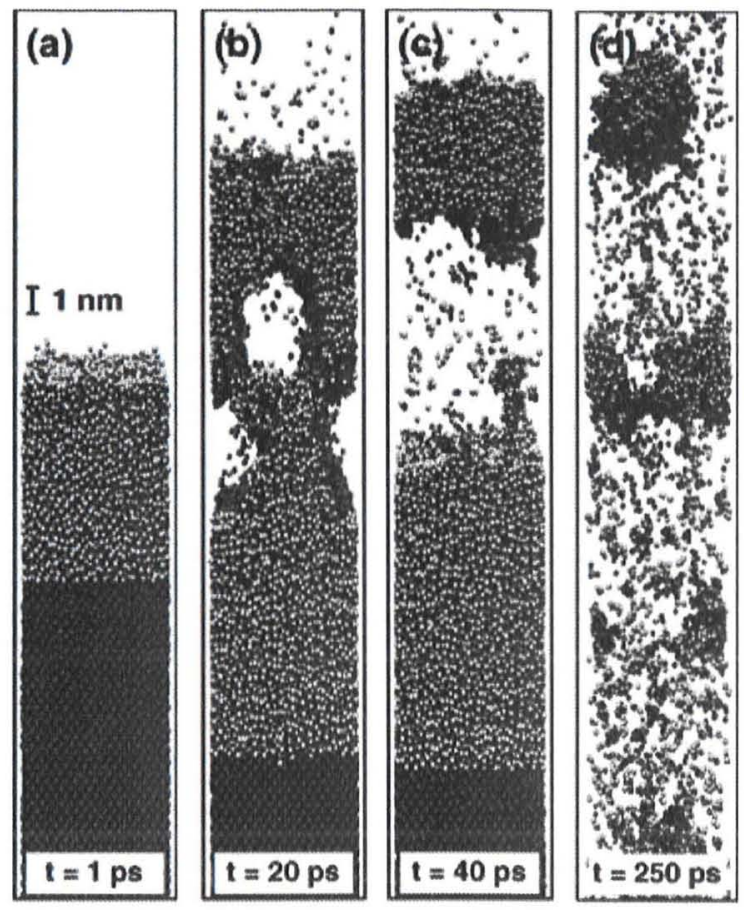

Figure 1 Visualization of molecular dynamics simulations showing snapshots of atom coordinates during the ejection from a silicon surface at different pulse durations of light pulses at 266$\mathrm{nm}$ wavelength: a-c) at a fluence of $375 \mathrm{~J} / \mathrm{m}^{2}$ for $500-\mathrm{fs}$ pulses

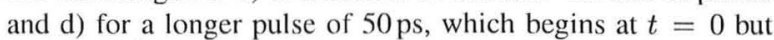
at $550 \mathrm{~J} / \mathrm{m}^{2}$. The dark balls mark crystalline silicon, the light grey particles are in a molten region. During picosecond ablation the material shows a larger amount of melting and vaporization. Reprinted from [6] with permission from APS.

A central characteristic of the phenomenon is that the process is strongly fluence dependent, i.e. there exist sharp thresholds for the ablation process, which are linked to the phase transition [11]. These thresholds are specific to the very material and well known. The ablation threshold for gold is found above $4000 \mathrm{~J} / \mathrm{m}^{2}$, for silicon above $1500 \mathrm{~J} / \mathrm{m}^{2}[11,12]$

Transparent materials can also be ablated when the electrical field strength surpasses the breakdown threshold [1]. Multiphoton and avalanche processes play an important role in the mechanism of light absorption. Dielectric breakdown for fused silica is observed above $2000 \mathrm{~J} / \mathrm{m}^{2}$ [1]. In this case multiple ionization and nonlinear absorption create a plasma. This plasma formation will become dominant for very short pulses in the femtosecond range. For metals the thermal ablation threshold will nevertheless be below the plasma-formation threshold [14].

Thermomechanical and photomechanical effects can also play a role in laser ablation [2]. Perez and Lewis [15] pointed out that ablation can also be induced by a mechanical effect, rather than a thermal phase transition. In that case the rapidly released thermoelastic stress can surpass 


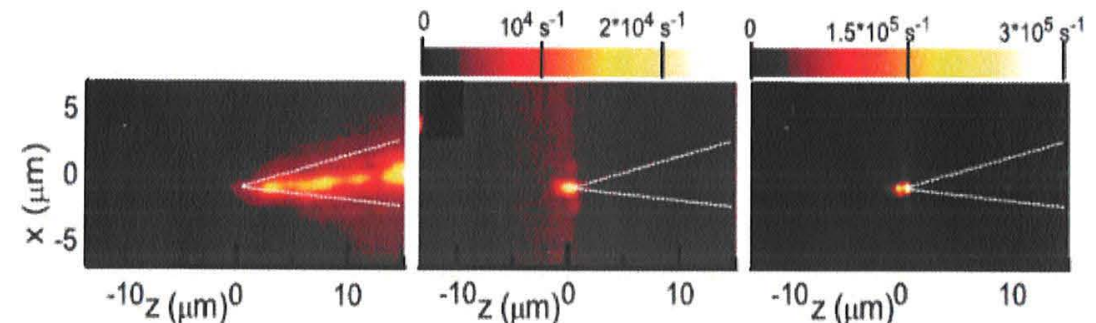

Figure 2 (online color at: www.lprjournal.org) Yicld of the 800-nm fundamental light scattering, nonlinear light emission, and photoelectrons (from left to right) when scanning the focused laser spot along a fine gold tip (dashed lines). Reprinted from [13] with permission from APS. the physical strength of the material during stress confinement, which leads to rupture and material ejection.

In essence, the thermal ablation method is characterized by a transfer of the deposited energy to the phonon bath and a description of the equation of state by means of defined temperature and pressure. Therefore, the phenomenology of ablation should not be different for any laser pulse that is shorter than the transfer of the energy from the electron gas to the lattice, as this transfer is the rate-limiting step with electron-phonon scattering times in the range of some 0.5 to 5 picoseconds $[16,17]$. The larger values apply for a strong excitation with a prolonged time span for complete energy transfer to the lattice [18]. The threshold fluence for ablation will also be independent of pulse length for sub-picosecond pulses as long as thermal dynamics is concerned.

In semiconductors an additional non-thermal pathway of phase transformation has been described, which allows for melting within a few femtoseconds $[19,20]$. This socalled non-thermal melting is ascribed to the massive excitation of charge carriers into the conduction band, converting the interatomic forces into antibonding states. It has indeed been observed that after rapid femtosecond excitation of silicon or germanium the lattice order disappears within about a hundred femtoseconds [21-23], which has been interpreted as an initial ballistic atom disordering due to the lack of remaining binding forces [23] or anharmonicity [24] This ultra-fast melting is considered as one important aspect in the ablation mechanisms of semiconductors as well.

\section{Tip-enhanced patterning}

The enhancement of an electrical field at a metallic tip is generally a well-known effect, which is explored in field ion microscopes such as the so-called 3D atom probe $[25,26]$ In this instrument a fine tip is analyzed atom by atom, by applying a strong electrical field to it, which extracts atoms from the surface. These are then resolved spatially by the projection of the flight path onto an imaging device to reconstruct the initial material composition. Efforts have been made to improve the field emission by additionally applying laser pulses to the tip $[27,28]$. Vella et al. $[29,30]$ have shown theoretically and experimentally that material can be ejected from laser-excited tips in an atom-probe configuration at low fluences. They connected the field enhancement also to an optical rectification process. In the same line the ablation from a flat surface can be enhanced and the fluence threshold can be reduced for very short femtosecond pulses. Dachraoui and Husinsky [31] have observed the ablation by means of changed pulse lengths and double-pulse experiments and discerned different regimes of electronic (or non-thermal) ablation, plasma-mediated ablation, and thermal ablation.

It has been shown that electron emission from curved surfaces follows tightly the field enhancement $[13,32,33]$, which has even been proven a valuable tool for the measurement of the near-field enhancement or even to control the field distribution by phase-shaped femtosecond pulses [34-36]. In Fig. 2 such an enhancement of both light and electron emission close to the apex of a sharp gold tip has been visualized. While the fundamental light scattering is seen all over the gold tip, the source for second-harmonic generation is located particularly at the tip, due to the nonlinear enhancement in the same way as photoelectron emission.

The selective excitation of resonantly enhanced modes at sharp tips such as in scanning probe microscopes promises to open new ways to structure materials by lasers below the wavelength-resolution limit. Nolte et al. [37] have demonstrated that a surface can be nanostructured by coupling intense femtosecond pulses into the fiber tip of a scanning optical near field microscope (SNOM). The improvement of this arrangement led to the development of the apertureless SNOM, in which a metal tip guides the applied light to the tip [38-40]. The first experiments which reported the manipulation of a surface by the coupling of intense nanosecond pulses to such metal tips, by Jersch and Dickmann [41], were simply explicable by thermal effects. A tip is brought close to a surface to be modified and a laser pulse is coupled to it. With a considerable thermal expansion the tip could be forced into the material, causing permanent structures [42-44]. Some very recent results indicate that a contribution to tip-induced structuring can be attributed to optical enhancement effects [45].

\section{Field enhancement near plasmonic structures}

\subsection{General Mie theory}

The electromagnetic response of dielectric or metallic particles can be described via Maxwell's equations in either 
analytical or numerical solutions. Mie [46] has given the first analytical result in spherical coordinates, i.e. for spherical particles. The response in absorption and scattering is developed in a series of multipole expansions taking the complex index of refraction as input. In the first (dipole) approximation the response of a particle much smaller than the wavelength $\lambda$ can be expressed as

$$
\sigma_{\mathrm{ext}}=\frac{9 V \epsilon_{\mathrm{m}}^{3 / 2} \cdot \omega \epsilon_{2}(\omega)}{c \cdot\left(c_{1}(\omega)+2 \epsilon_{\mathrm{m}}\right)^{2}+c_{2}(\omega)^{2}}
$$

with the spherical particle volume $V$, the speed of light $c$, the light frequency $\omega$, and the dielectric constant $\epsilon_{\mathrm{m}}$ of the surrounding medium. The functions $\epsilon_{1}$ and $\epsilon_{2}$ describe the real and imaginary parts of the wavelength-dependent dielectric function of the particle material $[47,48]$. The socalled surface plasmon resonance (SPR) is expected when the denominator of Eq. 1 becomes small, i.e. near $\epsilon_{2}(\omega)=$ $-2 \epsilon_{\mathrm{m}}$. This is the case for a number of metals, while for selected metals the resonance becomes very strong and sharp (silver, gold, aluminum, or copper [49]).

The SPR line is homogeneously broadened due to the ultra-fast dephasing of the oscillation of the conduction electrons, which has been clarified in a number of studies [50-52]. Furthermore, an inhomogeneous broadening in ensembles is caused by size and shape inhomogeneities [53,54]. Gold particles show as well a band due to interband absorption, which is present for wavelengths shorter than $520 \mathrm{~nm}$ [55]. A useful feature of the SPR is the dependence of the position and width on the particle size. The main effect comes from retardation effects of the light-matter interaction, when the particle size approaches the wavelength of light [49]. This size effect is seen as a red shift for increasing particle sizes and is important for particles above a diameter $D$ of $20-30 \mathrm{~nm}$. For larger anisotropic particles no analytical solution exists; instead, numerical approximations are used. Such methods, which do not need the symmetry of the system, are the finite difference time domain (FDTD) method [56] or the discrete dipole approximation (DDA) $[57,58]$. In the latter the object is divided by a fine grid (a few nanometers wide) on which interacting dipoles are located [59-61].

\subsection{Examples of spheres and triangles}

Some calculations of near-field distributions are shown in Fig. 3 for spherical particles. The full multipole series is included and the dielectric functions for gold have been taken from [47]. As the calculated gold particles are rather small, a dipole approximation for the plasmon resonance is still a good description. One observes that the plasmon resonance peak for $38-\mathrm{nm}$ particles is around $530 \mathrm{~nm}$ in water, while the limiting value for smaller particles tends towards $520 \mathrm{~nm}[49,62,63]$. The near-field distribution illustrates the effect that the enhancement is of dipolar nature along the polarization of the incoming light. As the oscillator strength is high, even at the shown wavelength of $400 \mathrm{~nm}$

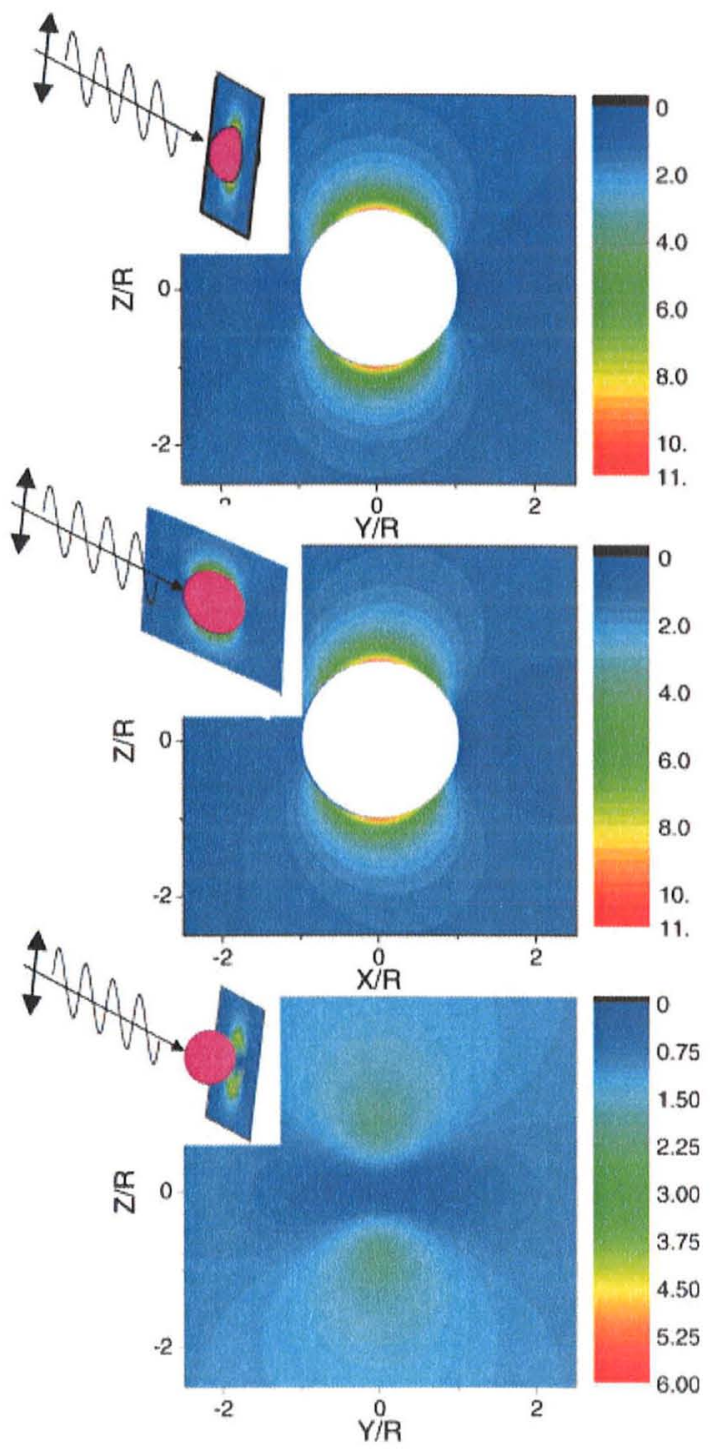

Figure 3 (online color at: www.lpr-journal.org) Calculated nearfield distribution $|E|$ around a gold nanoparticle with a Mie parameter of $\pi \cdot n_{\mathrm{m}} \cdot D / \lambda=0.397$ corresponding to a $D=38 \mathrm{~nm}$ gold sphere in water. Top: the electrical field distribution for a plane electromagnetic wave at $400 \mathrm{~nm}$ propagating in the $x$ direction, the $y-z$ plane is located in the sphere center. Middle: the field distribution in the $x-z$ plane and incoming light polarization in the plane. Lower: the field distribution in the $y-z$ plane, but in contact just behind the sphere. The color encodes in each case the field enhancement relative to the incoming wave.

(frequent-excitation condition for excitation with frequencydoubled femtosecond pulses from titanium-sapphire lasers), there is a local focusing of the field strength at the poles of the sphere, which amounts to about 11 times the incoming field. Right at the plasmon position this factor will amount to up to 40. The upper image shows the situation in a plane 

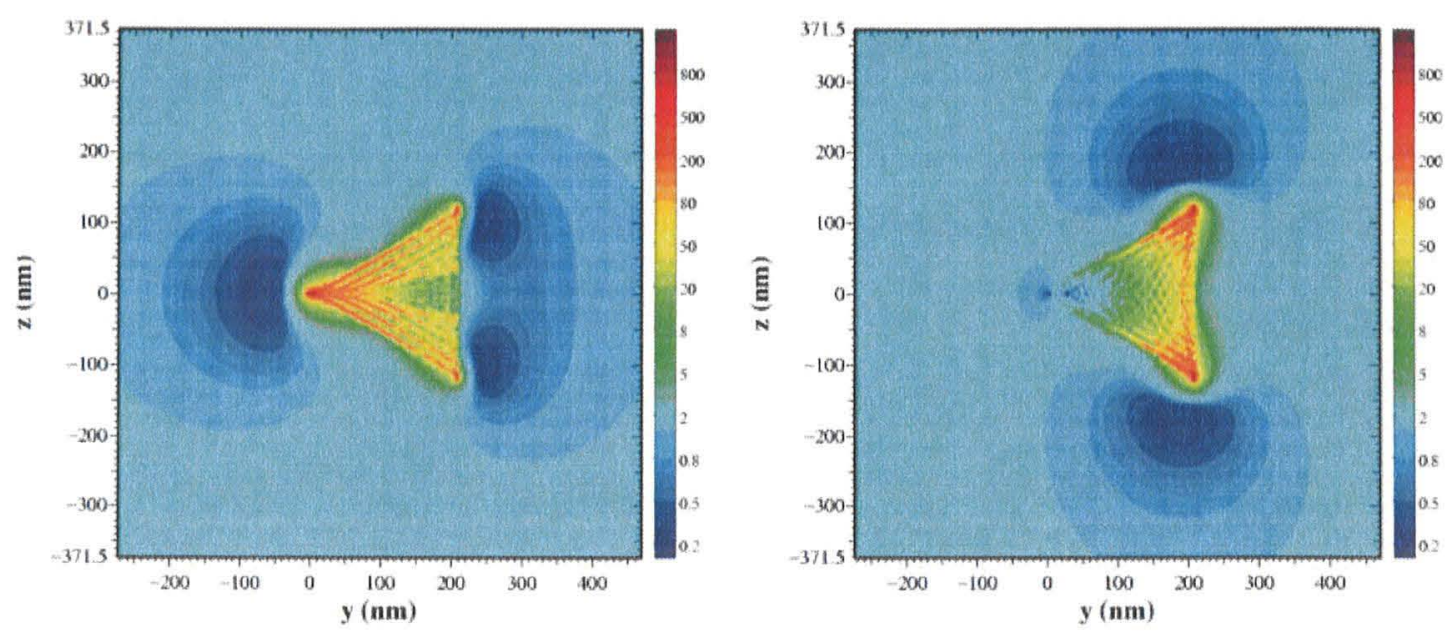

Figure 4 (online color at: www.lpr-journal.org) Calculated near-field distribution $\left|E^{2}\right|$ around a gold nanotriangle in the plane of the silicon substrate [61] for a laser polarization aligned horizontally (left) and vertically (right). The dielectric response of the substrate is fully taken into account. Reprinted from [61] with permission from Springer.

through the particle center perpendicular to the Poynting vector of the incoming radiation. In the second image a cut along the beam-propagation direction is depicted, showing that the dipole is already slightly distorted towards the beam direction. This distortion will increase with particle size, showing the effect of the higher multipole contributions to the Mie series. Finally, at even larger particles the focusing in the forward direction will merge with geometrical optics, identical to a geometrical focusing condition. This focus has been well employed to drill small holes in a substrate by irradiating dielectric spheres attached to the substrate $[64,65]$.

Finally, the lowest image in Fig. 3 shows a field distribution behind the particle in a plane, which would be part of a substrate, which supports the particle when irradiating the structure at perpendicular incidence. The dipolar character is still preserved, while the enhancement is certainly lower, amounting for a factor of two. There is, however a non-analytical problem with supported particles. As the dielectric medium is no longer homogeneous, one would have to use the approximate methods for the calculation, which is not included in the shown data.

For more complex structures the above-mentioned numerical finite element calculations are used to show some similar effects. A triangle is an example of a non-trivial structure and relevant in the application of prestructured periodic nanostructures. The field is enhanced in a direction roughly parallel to the laser polarization (Fig. 4). The tips that are not oriented along the laser polarization do not experience such a field strength. The strongest enhancement builds up at points of strong surface curvature and the absolute value of the enhancement can largely exceed the spherical case due to the larger oscillating volume relative to the curvature.
Further modifications of the optical spectra are expected if particles come close to each other so that the resonances couple. An intuitive description uses the hybridization of modes [66-69], whereby parallel or antiparallel coupling of the modes of two adjacent particles causes red shifts or blue shifts, respectively, of the delocalized resonance. In practice, the red shift is most commonly observed in aggregates or coupled plasmonic structures [70]. The coupling bears an important consequence, in that the space between adjacent structures can see an increased field-enhancement factor due to the delocalization of the plasmon resonance [69]. Advantage of this effect is taken in surface enhanced Raman scaltering (SERS) detection of trace amounts of organic molecules near rough or structured metal surfaces. A manipulation of the interparticle distance and thus the evolution of the complex plasmon hybridization scheme have been demonstrated recently by approaching two triangles by the aid of an atomic force microscope (AFM) [71]. These so-called bowtie antennae are very efficient in coupling light in the interparticle space for near-field optical microscopes [72].

\section{Dynamics of near-field-induced ablation}

The field-distribution effects around nanostructures will dominate the structure evolution once the laser power is increased towards irreversible reactions. Kaemple, Podlipensky et al. [73-75] have seen a manifold of subtle effects on silver particles in a glass matrix, leading to a variable sample coloration and even dichroic optical properties. One determining structural motif turned out to be an elongation of initially spherical particles with fixed alignment to the laser polarization. This elongation could be either parallel or perpendicular to the electrical field depending on the 


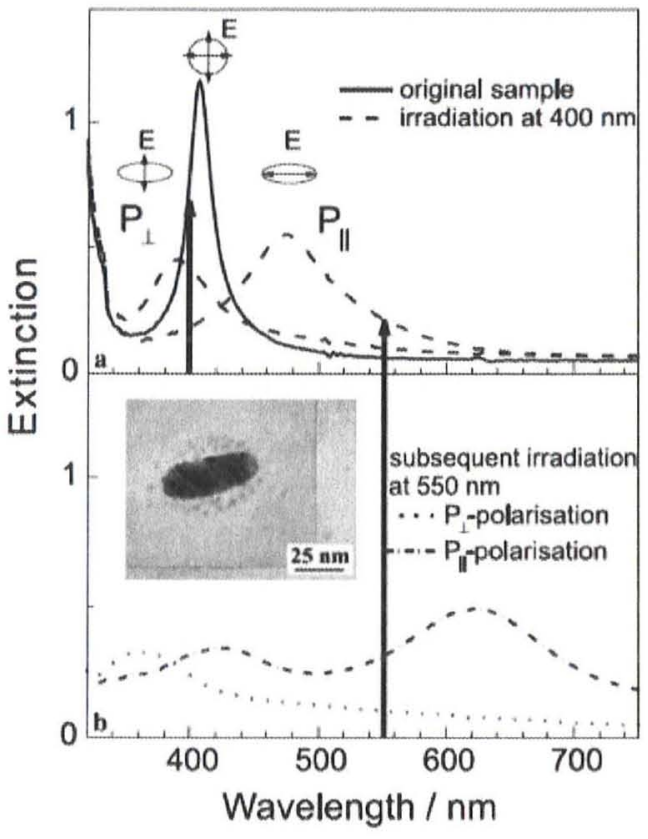

Figure 5 a) Optical extinction spectra of a silver nanoparticle containing glass before irradiation with femtosecond laser pulses at $400 \mathrm{~nm}$ and for two polarizations relative to the laser polarization. b) Further irradiation with a 530-nm laser beam enhances the plasmon peak shift observed in the first irradiation. The inset displays a TEM image of one particle with its aureole of smaller clusters. Reprinted from [75] with permission from Springer.

irradiation conditions (fluence and number of pulses). As most of the analysis was done post mortem, the mechanism could not be directly deduced. The polarization however pointed to the coupling of the laser field to the dichroic spectral properties of anisotropic nanoparticle ensembles.

The optical analysis of irradiated samples post mortem as well as in situ is particularly difficult, as the optical fingerprints of different structural motifs overlap in the same spectral region. The plasmon resonance of metal particles such as silver or gold is generally a good indicator for structurerelated properties, such as size, shape $[49,76,77]$, interparticle correlations [78], or lattice excitations [50,79-81] After irradiation of the embedded particles, Podlipensky et al. [75] found by transmission electron microscopy (TEM) that the initial particles were deformed and additionally an aureole of newly formed clusters appeared around them, which add to the spectral fingerprint. A corresponding TEM micrograph is shown in Fig. 5 together with the extinction spectra before and after irradiation. The plasmon position suffered a blue shift for a polarization perpendicular and a red shift parallel to the irradiation laser field. This is interpreted as an elongation of the particle in the direction of the electrical field and is found to happen on a time scale of hundreds of picoseconds $[82,83]$. This could be induced at a relatively low fluence of $500 \mathrm{~J} / \mathrm{m}^{2}$ and with repetitive excitation. In contrast, at larger fluences, even with a single pulse, anisotropy could be induced, shortening the parallel axis [84].

The Coulomb explosion of small clusters is an effect that is closely related to a non-thermal laser ablation process. In this case [85] the intense femtosecond radiation leads to massive photoelectron emission along the dipolar excitation, which destabilizes the ion attractive potential completely.

\subsection{Technical realization of a structural pump-probe experiment}

The understanding of the phenomenon of anisotropic structure formation is so far unsatisfactory as the kinetics and thermodynamics of the structural transformation are not resolved.

Ultra-fast time-resolved X-ray scattering experiments have been performed by using laboratory-based laser plasma sources $[21,86,87]$. They allow for an excellent time resolution but do not deliver by far the demanded $\mathrm{X}$-ray flux for the performance of scattering in disordered samples as in the case of nanoparticles. The latter has been realized at the synchrotron light source ESRF (European Synchrotron Radiation Facility), which delivers very intense and collimated radiation. The radiation is naturally pulsed due to the radio-frequency longitudinal acceleration of the electrons in the storage ring. These pulses have a temporal width of $50-150 \mathrm{ps}$ and a femtosecond laser can be locked on the time structure of the emission. At the beamline ID09B $[88,89]$ a femtosecond oscillator is phase stabilized to the bunch clock to a precision better than $5 \mathrm{ps}$, as sketched in Fig. 6. Its relative delay can be tuned by a phase shifter that changes the pulse-arrival time in steps of $5 \mathrm{ps}$ in the range of $11.8 \mathrm{~ns}$, the inverse of the repetition frequency of the laser oscillator (MIRA, Coherent Inc.) Larger temporal delays can be effected by amplifying a different chosen seed pulse in the regenerative amplifier (Hurricane, Spectra Physics). Thus in fact a large span in time delays can be realized, only limited by the repetition rate of the experiment, which was $1 \mathrm{kHz}$ in the present case as imposed by the laser system.

As the insertion device (undulator with $17-\mathrm{mm}$ period) in the storage ring emits at least at a frequency of $357 \mathrm{kHz}$ (corresponding to the round-trip time for electrons), the pulse train has to be diluted down to the $1-\mathrm{kHz}$ laser repetition rate. This is being done with a mechanical rotating wheel, which opens for at smallest 300 nanoseconds and runs in synchrony at $1 \mathrm{kHz}$. The X-ray beam is furthermore focused by a toroidal mirror [90] down to a $0.1-\mathrm{mm}$ focal size. Optionally, a monochromator can be used for high-resolution experiments, which are necessary for the measurement of the powder scattering of the excited gold particles to determine the lattice spacing. For time-resolved small-angle X-ray scattering (SAXS) [91] the radiation can be used without further monochromatization due to the inherent lower resolution necessary in the disordered sample. 


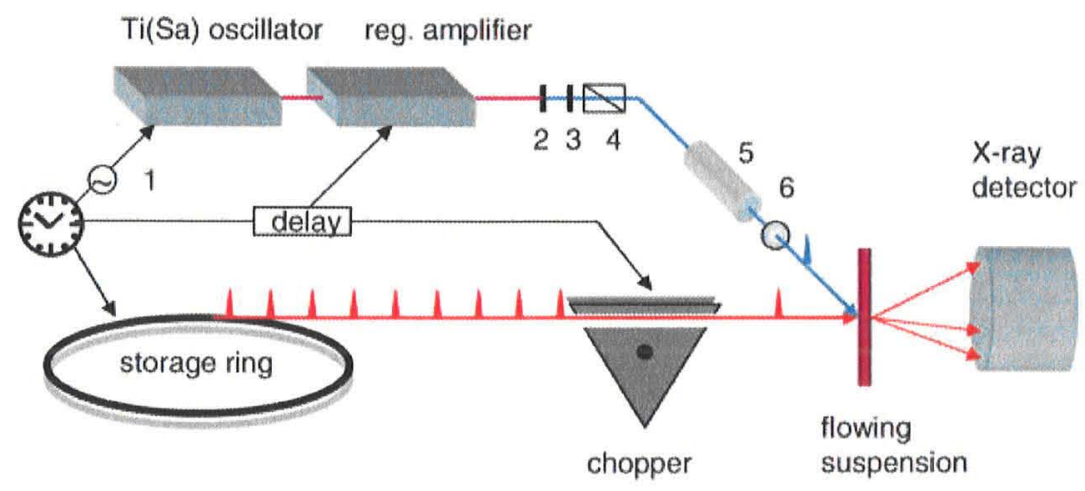

Figure 6 (online color at: www.lpr-journal.org) Experimental realization of the laser pump-X-ray probe experiment at the storage ring ESRF with the femtosecond laser being electronically coupled to the ring time structure by means of the bunch clock, a phase shifter (1), and delay units. The laser pulses coming from the oscillator-amplifier unit are frequency doubled (2) and led through a phase plate-polarizer combination $(3+4)$ for intensity control and then optionally stretched by propagation in a fused-silica rod (5). A lens (6) finally focuses the radiation onto the sample. The X-rays are shaped by an optional monochromator and focusing mirror (not shown). Selected pulses in synchrony to the laser emission are transmitted through the chopper. A 2D detector finally records the X-ray scattering.

The laser beam is frequency doubled in a nonlinear crystal and passed through a motorized combination of a half-wave phase retarder and a polarizer to scan the laser intensity. The effects of the short pulses are controlled by stretching the pulse length in a fused-silica prism from $100 \mathrm{fs}$ to about $1 \mathrm{ps}$. Finally, a lens focuses the radiation to a size of 2.5 times the X-ray size to ensure a homogeneous fluence distribution across the probed sample region.

Gold nanoparticles can be conveniently prepared by chemical synthesis methods in suspension. Some of these techniques such as the Brust-Schiffrin method allow producing a high density of particles, which are stabilized by surfactants, but are available only as small sizes below $10 \mathrm{~nm}$. The other historical method was developed by Turkevich et al. $[92,93]$ in the 1950s and allows us after some adaptations $[63,94]$ to derive well-monodispersed suspensions with tunable sizes. As these particles are charge stabilized, their density is limited to $1-2 \mathrm{mM}$ gold-ion content.

As the ablation process is irreversible, such an experiment has to be designed as a single-shot exposure. While the suspension is pumped at sufficient speed through an $\mathrm{X}$-ray capillary the pump (laser) and probe (X-ray) pulses hit a refreshed portion at each pulse pair.

By comparing the scattering pattern from the sample before the laser excitation and after excitation the pure difference signal due to the structure changes can be isolated, while external sources of scattering or stray radiation vanish. A post-mortem study of particles on a surface has been performed at the beamline CSAXS at the Swiss Light Source.

Particle arrays on a surface could be prepared either by self-assembly methods of presynthesized particles or by lithography processes. One elegant way is used frequently to form triangular structures of variable sizes and aspect ratios. This so-called nanosphere lithography uses preassembled polystyrene particles to evaporate gold onto the substrate through the thus-formed mask [95-97]. These structures have been used in a number of interesting photonic applications [98]. Surface-enhanced Raman scattering allows us to detect small traces of molecules that are located in the near field of these plasmonic structures, which boosts the probability for the Raman process by several orders of magnitude.

\subsection{Establishment of the thermal kinetics}

The experiments using pulsed X-ray scattering described in the previous section have measured the lattice expansion as a function of time and also as a function of fluence. It should be added that in the case of femtosecond excitation the fluence is a meaningful value rather than other intensity-related parameters, such as laser peak power, etc., as the two-temperature model for the electron and lattice sub-systems in metals predicts that heat is transferred from the electron gas to the lattice within the electronlattice equilibration time, which is much slower than the laser pulse length. Therefore, all energy is deposited before any structural reaction. The fluence is thus proportional to lattice temperature assuming a thermal equilibrium. The proportionality constant is solely determined by absorption coefficient and specific heat. For a time delay after laser excitation less than the characteristic cooling time (which ranges from 0.3 to $2 \mathrm{~ns}$ in the discussed range of sizes [99-102]) one can test the caloric relation between fluence and lattice expansion. It is indeed found that this relation holds to a very good level [103-105], which is seen in Figs. 7 and 8 in the uppermost graphs. For the suspended particles the agreement is quantitatively good. For surface-supported (free) particles the absorption coefficient is not known in the present case [106]. One problem here concerns the knowledge of surface coverage and distribution of interparticle spacing, which prohibits the calculation 


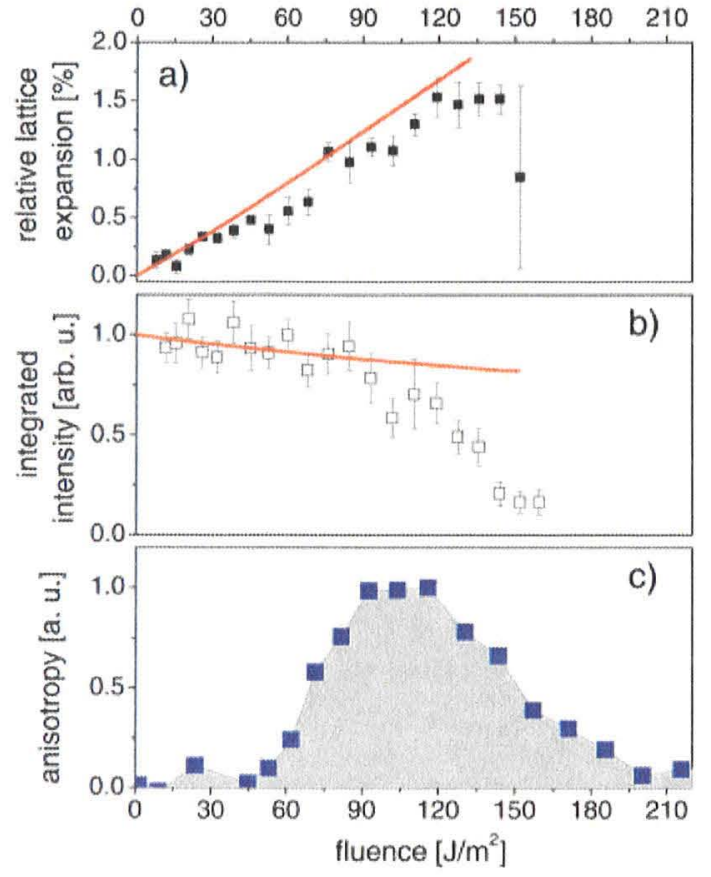

Figure 7 (online color at: www.lpr-journal.org) Determination of the lattice state in a flowing nanoparticle suspension (38-nm particles) at a 100-ps delay from the time-resolved powder scattering. a) The lattice expansion as a function of applied laser fluence (dots), the line is a calculation of the expansion from material parameters. b) Integrated intensity of the (111) powder ring as a function of fluence (dots), the line is a calculation of the DebyeWaller reduction of the scattering intensity. c) Anisotropy of the SAXS patterns indicating an anisotropic particle deformation.

of the single-particle extinction. The experiment itself can still serve to adapt this single parameter to again derive the proportional relationship of fluence and lattice temperature (shown in Fig. 8 for supported particles). Finally, the thresholds for particle melting can be determined using the temperature scale and the observation of vanishing of the Bragg peaks. While a reduction of scattering intensity is expected when raising the temperature (by the so-called Debye-Waller factor) a massive reduction is seen in the middle part of Figs. 7 and 8 at the melting fluence.

So far, the observations have been of regular thermal phenomena including laser heating of the particles and melting at the expected threshold. A particular anomaly occurs in Fig. 8 in the upper graph, where lattice expansion levels off lar below the melting point. This is, however, an artifact of the repetitive excitation during that experiment and a slow sintering with increased number of shots. The same experiment repeated with single-shot excitation shows regular expansion along the predicted line. The melting fluence is then determined at the point of the intersection of the thermal expansion versus fluence with the maximum possible expansion in gold, which amounts to about $1.8 \%$ [107].

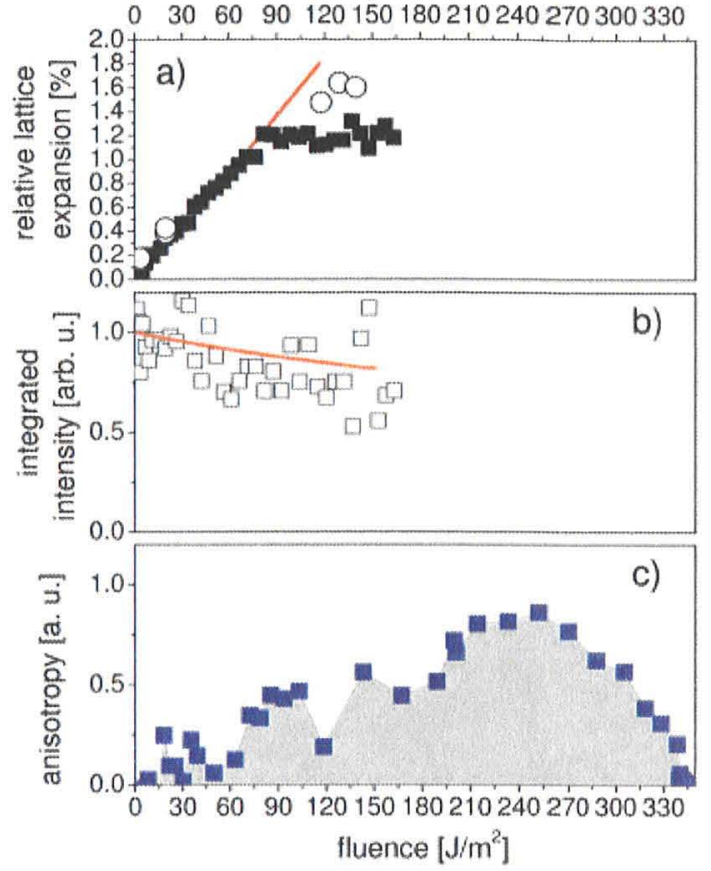

Figure 8 (online color at: www.lpr-journal.org) Determination of the lattice state of a particle monolayer on a silicon substrate at a 100-ps delay from the time-resolved powder scattering. a) The lattice expansion as a function of applied laser fluence (dots), the line is a calculation of the expansion from material parameters. The change of expansion with fluence at around $90 \mathrm{~J} / \mathrm{m}^{2}$ is due to an in situ changing absorption coefficient during sintering, which is absent in single-shot excitation (open circles). b) Integrated intensity of the (111) powder ring as a function of fluence (dots) the line is a calculation of the Debye-Waller reduction of the scattering intensity. c) Anisotropy of the SAXS patterns indicating an anisotropic particle deformation.

In order to really identify the mechanism of material transformation one needs to establish procedures to characterize the structural state of the sample, such as the lattice state to high spatial and, speaking of short-pulse phenomena, as well to high temporal resolution. This has been done first with optical methods $[19,20,109]$, which are, however, limited in the interpretation of melting, in particular on the nanoscale [80]. Experiments with plasma-produced Xrays [21] have been quite successful for the analysis of single-crystal surfaces and their melting properties on a sub-picosecond time scale. However, on a nanoscale the inherent disorder in the system and the small amount of material prevent the application of this method, albeit it offers up to now the highest time resolution. Ultra-fast electron diffraction (UED) [110] has been used for ultra-fast structure determination of photoexcited species such as molecules [111], nanoparticles [108], or films [16, 112]. The time resolution for UED can be in the range of one picosecond and the high scattering cross section facilitates the detection of the nanoparticle signal. 

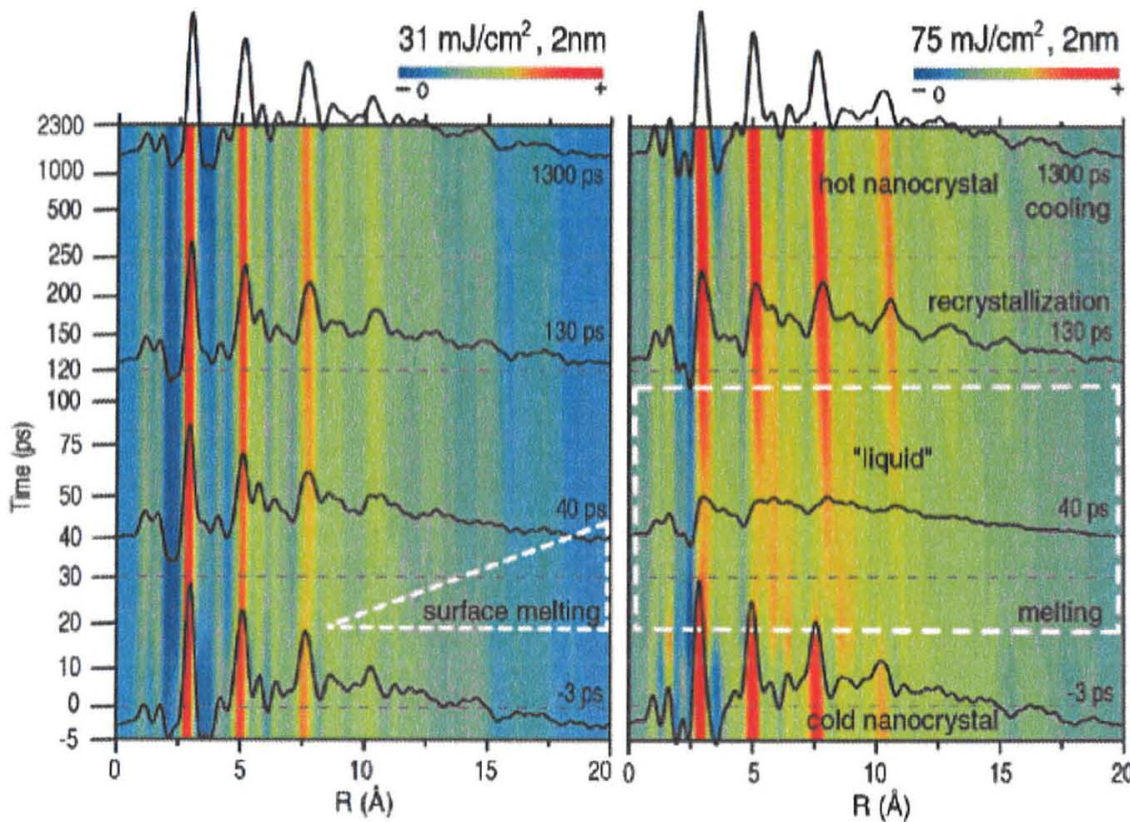

Figure 9 (online color at: www.lprjournal.org) Real-space transformation of electron-scattering data on laser-excited gold nanoparticles into the atomic pair correlation function. The melting at the higher fluence is seen on the right-hand side as a disappearance of all the ordered lattice planes, while at the lower fluence only the long-range correlations are reduced, which is interpreted as surface melting. Reprinted from [108] with permission from ACS.
Ruan et al. [108] have probed the lattice state of femtosecond laser excited gold particles on a surface by pulsed electron scattering using a laser-triggered photocathode. The accessibility of several Bragg reflections (powder ring) for the particle ensemble even allowed transforming the scattering data into real-space atomic correlations (see Fig. 9). The lattice expansion is a direct indicator for phonon and thermal excitation. They could reproduce some earlier reported findings on vibrational excitation and cooling kinetics $[100,103,113]$. In addition to that, the realspace data gave further hints of even more subtle structural phenomena, such as surface melting.

\subsection{Ablation and nonlinearity}

While the lattice state is mainly an indicator for the thermal excitation the morphological changes, such as ablation, are visible by shape changes. In a post-mortem analysis this would be visualized by real-space methods such as electron microscopy or atomic force microscopy. Here we deal with dynamical phenomena which favor an in situ probe, such as time-resolved small-angle scattering. It has been applied to the gold-particle suspension in the described pump-probe scheme [91].

The difference in scattering at very low momentum transfer $Q \simeq 1 / D$ with the system dimension $D$ (e.g. the particle size) is a direct fingerprint of the size or shape transformations. It has been observed that the excitation by a single laser pulse can induce a drastic difference signal [114]. This signal will show areas of intensity increase or decrease, which in a narrow-fluence region exhibits an anisotropic pattern. This anisotropy is shown to be aligned with the laser polarization (see Fig. 10). It should be recalled that the
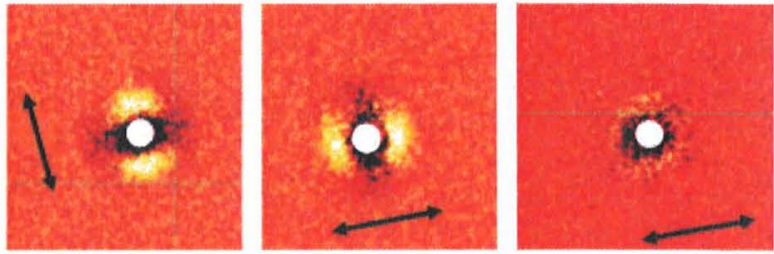

Figure 10 (online color at: www.lpr-journal.org) Difference scattering patterns of a gold-nanoparticle suspension of $38 \mathrm{~nm}$ at $2 \mathrm{~ns}$ after the femtosecond laser flash as measured by SAXS. The scattering patterns only differ by the laser polarization (left to middle) and the pulse length ( $100 \mathrm{fs}$ for the left-hand and middle images, $1 \mathrm{ps}$ for the right-hand image), but not the laser fluence, which was all in three cases $95 \mathrm{~J} / \mathrm{m}^{2}$.

particles are initially spherical. If after the reaction either the single particles remain spherical or the ensemble of anisotropic particles is randomly oriented, only symmetrical modifications of the scattering would be expected. We see two lobes of positive intensity in the direction of the laser polarization accompanied by a decrease in the center and a relatively unchanged meridian perpendicular to the laser polarization. The explanation for this pattern is a reduction in size of the particles from a native sphere to a rotational spheroid, where the axis along the laser polarization is reduced in size. A reduction in real space causes a widening of the scattering function in reciprocal space (in the Guinicr region $[115] Q<\pi / R$, with $R$ being the particle radius). Thus, it induces an intensity increase in that direction, while due to the reduced total mass of the particles the forward-scattering region (center of the images in Fig. 10) shows an intensity reduction. The direct 
conclusion is that the axis of size reduction is along the two lobes of photoinduced intensity increase in the images in Fig. 10. This finding is confirmed by the calculations of the scattering modification for a transition of a sphere into an ellipsoid. The amount of change can be quantified by the scaling to the total scattering of a particle; a $20 \%$ reduction of the axis parallel to the laser polarization is deduced.

This finding of a particle modification in the laser field is still not very instructive, as long as the very mechanism is unclear. One could imagine a kind of "hole burning" effect of selectively molten particles in an inhomogeneous ensemble, or a semielastic response of the surface energy after melting or surface melting. The first check is the tuning of the pulse length of the laser radiation. A propagation of the pulses through a 300-mm silica rod caused a stretching from an initial 100-fs pulse to almost $1 \mathrm{ps}$. The latter is still shorter than the electron-phonon equilibration for highly excited particles and should therefore not impact on any thermal phenomenon. Despite this, the stretching causes the observed anisotropy almost to disappear, showing the nonlinear and in this case non-thermal origin of the structure-formation process (rightmost image in Fig. 10). In fact, the electron-lattice coupling is the same for both pulses, but the electrical field of the laser pulse changes according to the pulse length, i.e. it is decreased by a factor of 10 upon pulse stretching. For deducing the field strength at the particle surface, we make use of the Mie equations by calculating the enhancement described in Sect. 4 . The field within the duration of the laser pulse is then found to approach values of $1.2 \times 10^{10} \mathrm{~V} / \mathrm{m}$. Adding an optical rectification effect at the interface, Vella et al. [30] even derived up to $10^{11} \mathrm{~V} / \mathrm{m}$. This is clearly a magnitude which surpasses reversible interactions. It had been shown that material transport can be induced at these levels, either in field-emission setups or in laser-excited nanoscale tips with applied electrical fields [27, 28].

This tells us that the particles are shape transformed by massive material ejection from the poles of the spheres with the electrical field of the laser pulses as driving force. This is postulated as an ablation process. Even more informative is the inclusion of the known thermal conditions of the different aspects of excitation explained above. The observation of a structural anisotropy is limited by two thresholds. The lower threshold, at which the ablation process sets in, may be related to a threshold in field strength, but interestingly is close to the bubble-formation threshold in the surrounding liquid $[104,116]$. The second threshold, upon which the anisotropy disappears on increasing the laser fluence, is located at the melting point of the particles as seen by the comparison with Fig. 7. This gives a proof that during the ablation process the particles are still in a solid state and the deformation process is not connected to a melting scenario. When surpassing the melting threshold, ablation still occurs, but the relaxation of the liquid drops to spheres hides the anisotropy of the process.

We explain the lower threshold, which coincides with the bubble-formation threshold, by the restriction of the liquid rather than a classical ablation threshold. In classical ablation from solids the threshold is normally connected to the explosive boiling of the ablated material $[8,9]$ or plasma formation [1]. In our case an effective material transport can only occur if the surrounding liquid is removed by the formation of bubbles.

\section{Irradiation experiments on particles on surfaces}

While particles in suspension show a very well traceable structural transformation it is more appealing to regard surfaces with nanoscale resonating structures on them. Gaining control over the laser-induced transformations of lateral structures, one obtains interesting tools for nanomachining. Both sub-wavelength features are achievable as well as a parallel patterning process in self-assembled structures.

\subsection{Spherical particles}

The first approach has been to use large dielectric colloids as microlenses to pattern the underlying substrate [118,119]. On reducing the size of the colloids below the wavelength of light the supposed single hole (through geometrical focusing) develops into a dumbbell structure with clear anisotropy, as seen in Fig. 11 [117]. The mechanism is readily explained by the dipolar lobe of near-field enhancement even without plasmonic excitation. The enhancement still amounts to a factor of two, which is within the same order of magnitude as the off-resonant excitation of gold spheres as calculated in Fig. 3. Nedyalkov et al. [120] and Eversolc et al. [121] have irradiated single gold particles on a silicon surface and seen similar two-lobe structures.

The initial particle has been destroyed completely and additionally holes have apparently been carved into the substrate, as shown in Fig. 12. The near field has consequently induced damage to the underlying substrate. As these studies are done post mortem, there is no direct connection to
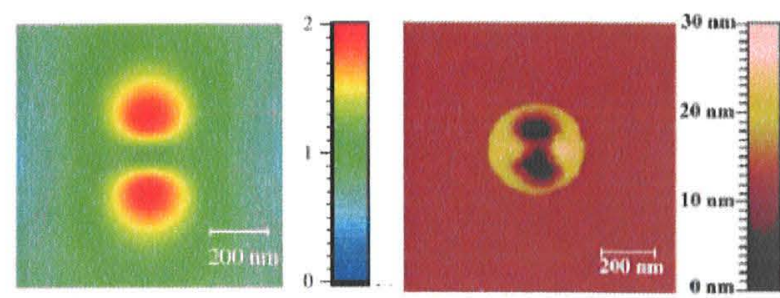

Figure 11 (online color at: www.lpr-journal.org) (Left) Field distribution $|E|$ below a spherical particle in the plane of the substrate as false-color distribution. The field enhancement relative to the incoming field amounts to a factor of up to 2.1 ; the effect of the substrate is neglected. (Right) Topographical image from the ablation crater below such a spherical particle as determined by an AFM. Reprinted from [117] with permission from AIP. 

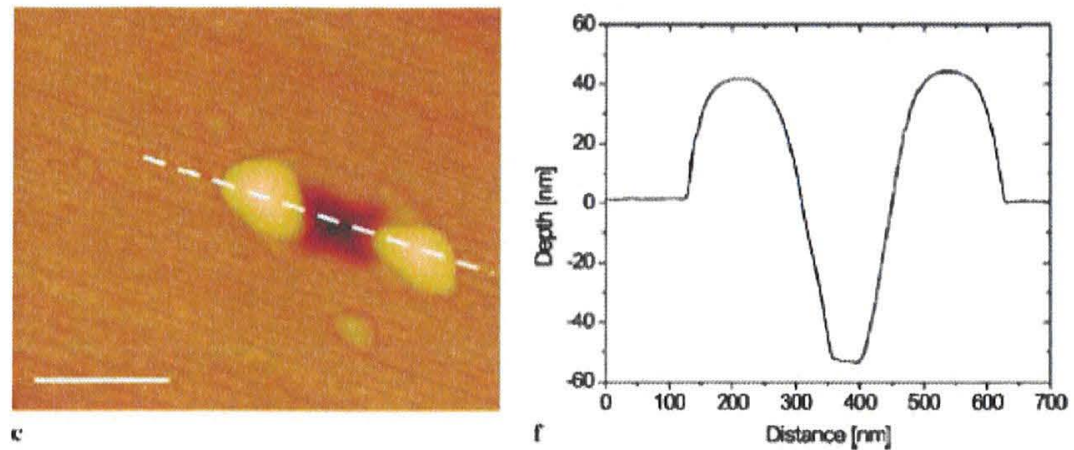

Figure 12 (online color at: www.lprjournal.org) Surface topography of a silicon surface with an isolated gold particle after irradiation with a femtosecond pulse at $780 \mathrm{~nm}$ with a fluence of $1280 \mathrm{~J} / \mathrm{m}^{2}$ together with a height profile on the right. The scale bar on the left is $200 \mathrm{~nm}$. The elevated structures are debris from the gold particle. Reprinted from [121] with permission from Springer.

the thermal kinetics of the substrate. Despite this, advantage has been taken of the fact that the ablation threshold of silicon is a well-known quantity and can be identified in the experiments if a Gaussian-shaped laser profile is applied onto the sample. The ablation threshold will be spatially identified by an ablation crater which is sharply encircled by the isointensity line at the threshold fluence. In [121] the threshold is derived to be $1910 \mathrm{~J} / \mathrm{m}^{2}$. At the same time, ablation beneath the gold nanoparticles occurs at a fluence of $82 \mathrm{~J} / \mathrm{m}^{2}$ at optimal polarization. Further analysis shows that this ablation process also bears a nonlinear component, as the threshold scales with the peak electrical field rather than with the deposited intensity. Eversole et al. [121] showed in a very didactic way that by tilting the laser incidence angle with respect to the surface one pole of the near field points downwards onto the surface, which increases the field enhancement in the surface plane to a factor of 24.

A similar process is being used to drill holes into a flat substrate. Nedyalkov et al. [120, 122, 123] have employed larger particles where the multipole contributions to the Mie resonances are more evident to form a forward focusing of the near-field intensity mainly located below the particles, if the laser impinges perpendicularly from the top of the structure (see the sketch in Fig. 13). This effect converges to the classical limit of focusing spherical lenses when increasing the particle size further, as demonstrated by polymeric spheres from patterning surfaces $[2,119,124]$. In this limit the application of relatively long laser pulses

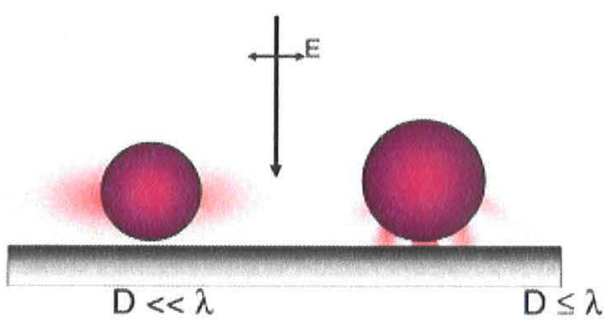

Figure 13 (online color at: www.lpr-journal.org) Development of the field distribution from very small particles (diameter $D \ll \lambda)$ on a surface to increasing size ( $D$ approaching $\lambda$ ) and consequently additional multipole resonances, which focus more light directly below the particles. from nanosecond sources would still result in a patterning of the surface [125-128], while only femtosecond lasers have high enough electrical fields to cause ablation even for small structures, which then display the dipolar features [121, 129].

In most of the above cases the particles are completely destroyed and removed from the surface, while only the indentations on the surface and some residual material are left behind. Clarity about the origin of the residuals can be obtained by selective etching of organic or metallic parts of the surface and repetitive imaging. The structures observed in Fig. 11 are identified as partly oxidized silicon redeposited at the elevated rims.

The kinetics of the structural transition can be clarified in a similar way as for the gold spheres in suspension as presented in Sect. 5.2. A single-shot excitation can be realized by a fast displacement of the substrate under the repetitive laser excitation. As the substrate has to be homogeneously covered by gold particles a self-assembly process has been chosen to deposit a submonolayer coverage of particles on a glass substrate $[78,130]$. One peculiar issue with this self assembly is that the particles are positioned randomly on the surface with a finite probability to touch each other. For a method that probes the ensemble average this has the consequence that a plasmon coupling can exert forces on the particles and also the positional correlation. Both motifs are seen in structural methods such as SAXS [106].

For a SAXS pump-probe experiment as discussed in Fig. 14 we come to the conclusion that the irradiation process of small gold particles on a surface leaves behind an anisotropic structure. The thresholds are comparable to the irradiation of gold particles in water, as seen by the comparison of the anisotropy and lattice expansion as a function of laser fluence. In Fig. 8 the thermal lattice expansion is compared to the anisotropy in the scattering image as an indicator for nonlinear ablation. Compared to the relevant result in suspension in Fig. 7, the anisotropy persists to higher lattice temperatures, where the particles are presumed to be melted. The scattering pattern, while also showing an anisotropy as before, is qualitatively different from the counterpart in suspension. The seemingly inverted contrast is a result of the close positional correlation of adjacent particles. The laser field provides a repulsive force to particles that are oriented along the field polarization, 


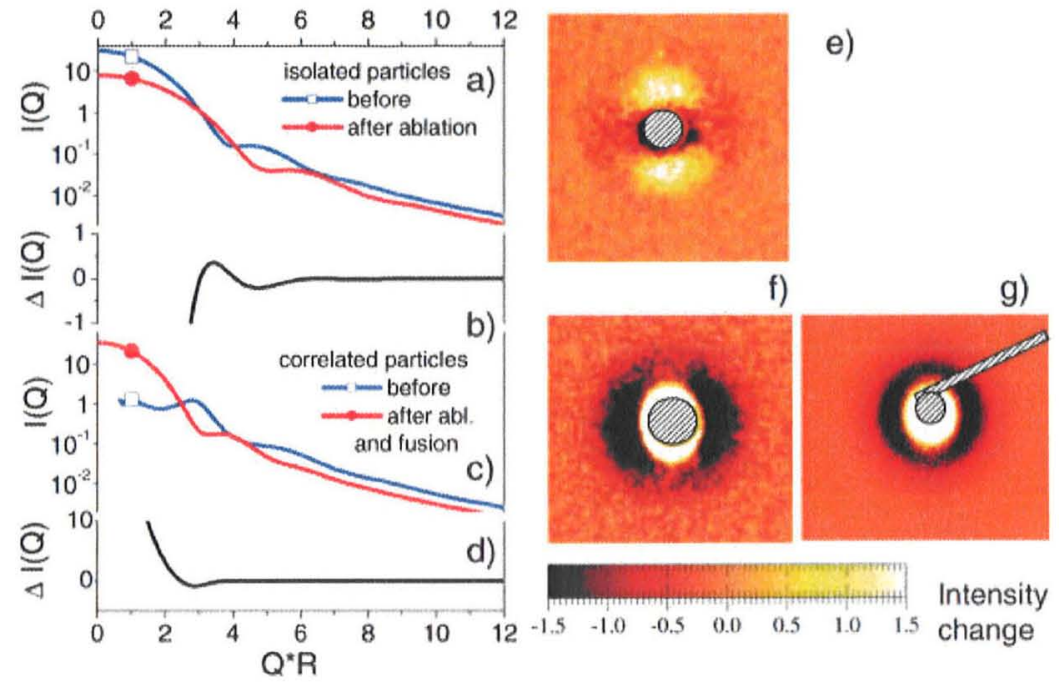

Figure 14 (online color at: www.lpr-journal.org) Simulation of the SAXS scattering cross section $I(Q)$ along a scattering vector along which the morphology of the particle is changing. The uppermost plot a) shows a decreasing particle radius and below the resulting difference scattering b). The third plot c) depicts the change of the scattering function from correlated particles on a surface to isolated ones after fusion (which increases the particle size). The lowest frame d) finally shows the difference scattering from this modification. While for isolated particles a decrease in scattering intensity is observed for low $Q$, an increase will be observed for the supported particles. Difference scattering patterns (2-ns delay) of a gold nanoparticle suspension of $38 \mathrm{~nm}$ e) compared to a similar setup of single-pulse excitation of particles on a glass surface at a delay of $100 \mathrm{ps}$ f). For comparison, the post-mortem analysis is shown in the right-hand image g). The hatched area is inaccessible due to the blocked direct beam $(Q=0)$.

while they can fuse perpendicularly to the field polarization when the melting point is approached. The similarity of the ultra-fast data $100 \mathrm{ps}$ after laser excitation with the corresponding post-mortem analysis in Fig. 14 supports this finding [106].

The corresponding effect is shown in Fig. $14 \mathrm{c}$ and d, with a correlation peak at $Q \times R \simeq 3$ that disappears after excitation, while the scattering for small $Q$ increases due to the separation of the particles. This is caused by the initial suppression of the forward-scattering intensity for closely positioned particles, which becomes less important after excitation. The resulting change in scattering is shown together with the full scattering before and alter laser excitation and matches the regions of intensity increase and decrease in the $2 \mathrm{D}$ difference maps along a radial coordinate from the center of symmetry (Fig. 14c-g).

As bottom line, laser irradiation of particle-covered surfaces allows us to create anisotropic structures both below and above the particle melting point. Near-field ablation and the modulation of the particle-fusion process by near-field forces both play a role in the morphology modification.

\subsection{Advanced geometric structures}

There is a strong interest in tailored particles from the application point of view, where field anisotropies can be tuned by the shape. The field enhancement can be much higher than for spherical particles. Nanorods or nanotriangles serve as antennae for the concentration of the radiation. In order to understand the field distribution a method had to be employed to experimentally verify the local field. Photoemission electron microscopy (PEEM) has been successfully used to image the field-enhanced photoelectron emission around arrangements of nanostructures in both space and time. The ablation, on the other hand, can also be used as a very local tool to imprint the near-field pattern onto the underlying surface. From a practical point of view this represents a template for the structuring process $[124,131]$ in parallel fashion as compared to a sequential structuring as for AFM-tip patterning or electron-beam lithography. Here the attempt can be made to use the ablated pattern as a "photographic" negative for the imaging of the peak field distribution at irradiation.

When looking at these triangles one can indeed observe pronounced holes in two of the three corners after irradiating the structure with a femtosecond pulse at $800 \mathrm{~nm}$. At a first view this agrees well with the calculated fields (Fig. 4). The size of the holes is seemingly much smaller than the wavelength of light and compatible with the range of the near-field enhancement of a couple of nanometers. One should nevertheless be aware that there may be a resolution limitation in this "imaging" process, which scales with the size of an ablated spot even at delta-like excitation. Nor is it a linear process, as already explained. The experiment nevertheless shows that this material-related resolution allows for the imaging of nanometer-sized field distributions. 

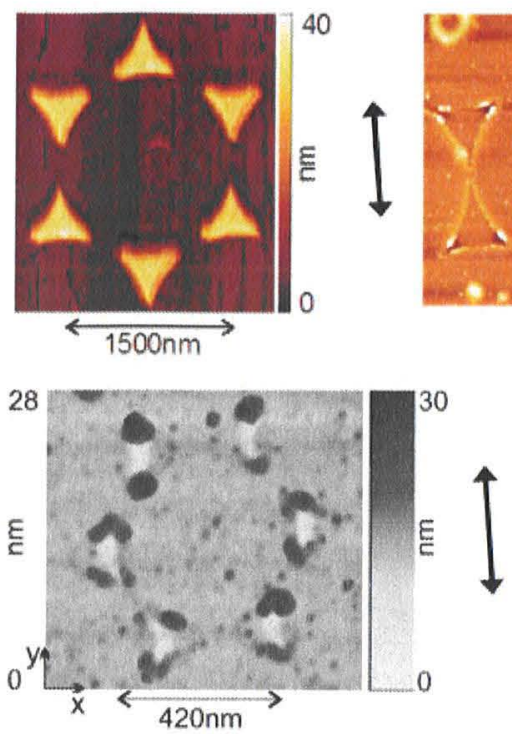
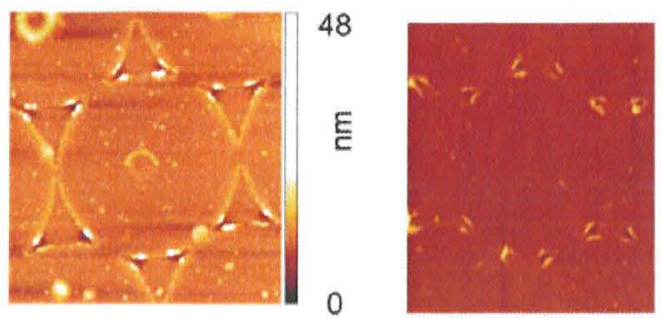

Figure 15 (online color at: www.lpr-journal.org) (Top) AFM images from an array of large gold nanotriangles formed by nanosphere lithography from 1500-nm polystyrene colloids. The left-hand image shows the native periodic pattern, while the middle image is taken after single-shot laser irradiation. On the left-hand side the residual gold contamination has been etched away, leaving behind the ablation crater. The ablation pattern is oriented perpendicularly to the laser polarization (arrow). (Bottom) AFM image of the ablated structure from smaller triangles that are templated by $420-\mathrm{nm}$ colloids. The ablation is aligned with the laser polarization. The gold thickness was $25 \mathrm{~nm}$ in each case. Reprinted from [117] and [61] with permission from AIP and Springer, respectively.
Looking closer at the comparison between experiment and simulation, an astonishing difference can be observed (Fig. 15, top). The array has been irradiated with the laser polarization along the dimeric orientation of a triangle pair. Here the field enhancement would naturally be expected to appear in the space between the two adjacent tips. This would be the consequence of a delocalization of the plasmonic excitation on the particles as coupled oscillators. Despite this, no ablation is visible at all within this gap, but instead at the far edges of the triangles. After an etching process (right-hand image) the holes in the substrate are more easily identified. This behavior is not reflected by any calculations of isolated triangles as described in Sect. 4. On the other hand, it has been said that conceptually new phenomena arise when plasmonic particles couple electronically. Here we see one consequence that the near field shows a more complicated structure. Indeed, a repetition of the experiment at an isolated triangle harmonizes the situation, the ablation patterns and thus the field enhancement appear now along the polarization direction. As the triangles are far apart compared to the distances of the near fields, one can exclude near-field coupling. This happens as well with smaller structures, which are further below the wavelength of light. As depicted in Fig. 15, bottom image, at these three times smaller structures the ablation craters are aligned in a dipolar fashion along the laser polarization. Therefore, it was proposed that the periodic arrangement of the particles leads to efficient diffraction [61]. The diffracted wave interacts with the incoming wave and introduces an inhomogeneous excitation of the triangles. This interpretation is further strengthened by the experimental observation of near-field distributions that do not appear in the simulations. There, the general lateral distribution does not change with size of the particles while in the experiment near-field enhancements in the middle of a triangle side appear [117]. Thus, it can be concluded that the periodic arrangement of plasmonic components may change the near fields.
A great variability lies in the interaction of light fields with complicated nanostructures. This should give more freedom of intentional manipulation of the nanostructures, but also allows us to establish tools to understand these non-trivial reactions by stepwise increasing the level of complexity and imaging the near field.

A study by El-Sayed and coworkers $[132,133]$ has more deeply examined the fate of the gold triangles of similar periodic structures at fluences below the modification of the substrate. They have found a very subtle dependence of the particle dynamics on the wavelength of the laser excitation. When irradiating with 400-nm light far away from the (coupled) plasmon resonances the gold triangles just start to melt and finally relax to spheres when dosing the light in multiple laser pulses. The situation changes drastically for 700-nm or 800-nm laser light. The particles start to lift off the substrate and fly completely unmodified off the support. While this has been explained by some evaporation effects, it is appealing to relate the lift-off process to the near-field enhancement close to the substrate. Thus, this effect also puts a handle on the forces between the particles and/or the substrate.

\section{Conclusion}

Near fields are present in any nanometer-patterned structure and should be properly taken into account in order to understand the interaction with intense laser pulses. This near field amplifies any laser effect. In particular, resonant interactions such as plasmon excitations boost the nearficld amplification of the clectromagnetic field. Plasmonics of nanostructures is a fascinating field, which unites sub-wavelength phenomena with nonlinear material-light interaction. It is worth the effort to understand light-matter interactions more deeply as it gathers non-trivial but at the 

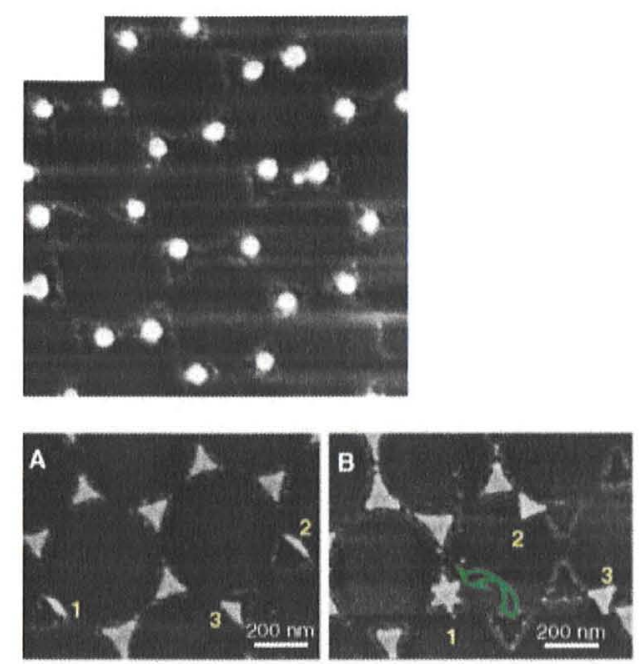

Figure 16 Electron micrographs from irradiated nanosphere lithography structures with different wavelengths of the laser light. At the upper image $400-\mathrm{nm}$ light was used at $3 \mathrm{~W} / \mathrm{cm}^{2}$ for 180000 shots equal to a fluence of $30 \mathrm{~J} / \mathrm{m}^{2}$ [132]. The heating leads to partial melting and dewetting from the substrate. The lower two images were taken after single-shot excitation with $800-\mathrm{nm}$ pulses at $41 \mathrm{~A}$ ) and $51 \mathrm{~B}) \mathrm{J} / \mathrm{m}^{2}$ [133]. Here the wavelength of the light is close to the plasmon resonances and thereby exerts large forces on the tips of the triangles due to the field enhancement. Reprinted with permission from ACS.

same time educative description. It is also of imminent importance for present-day photonic applications, which make use of the nonlinear type of interaction. Surface-enhanced Raman spectroscopy is one of the fields, but plasmonic structures in general serve in a number of applications as probes for very short ranged interactions and structural features, e.g. in biophotonic applications. Amongst the efforts to understand and use the field-dependent phenomena are non-reversible laser patterning methods, which both use and create field-enhanced structures. We have shown that understanding of the laser-induced reactions can be gained to a deeper level by resolving the structural dynamics of the laser-excited structures and comparing them to the thermal kinetics. The near-field-induced ablation process is observed to modify the morphology of the excited particles and also a nearby substrate. It is a nonlinear (and as well a non-thermal) effect that can be well correlated with the field distribution in these nanostructures. Classical limitations in feature sizes of optically induced structures can be overcome easily by utilizing the near fields in a nanoscopic environment. The near field decays typically within tens of nanometers, restricting the impact of the laser modification to this length scale.

Acknowledgements We express our gratitude to collaborators V. Kotaidis, A. Siems, O. Schubert, and J. König-Birk, who helped with some of the experiments. We thank M. Wulff and Q. Kong for the assistance at the beamlines ID09B (ESRF) and A. Menzel at the beamline cSAXS (SLS) and ESRF and SLS for support. This project was funded by the DFG through SFB 513 and the Center for Junior Research Fellows Konstanz.

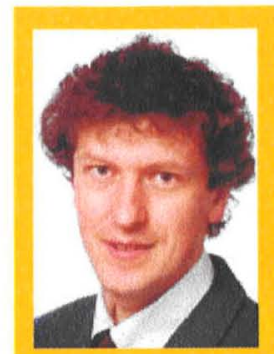

Anton Plech received his diploma and $\mathrm{Ph}$. D. at the Ludwig-Maximilians University in Munich. After a re search stay at the European Synchrotron Radiation Facility (ESRF) in Grenoble (France) he joined the Physics Department at the University of Konstanz as leader of a Junior Research group, where he received his habilitation. Since 2008 he has been a scientist at the Institute for Synchrotron Radiation within the Karlsruhe Institute of Technology. His main interests are dynamics of condensed matter, particularly nanoparticles. He is working on time resolved methods both in ultrafast laser spectroscopy and pulsed X-ray scattering. In 2004 he received the "Young Scientist Award" at the ESRF. $\mathrm{He}$ is an author or co-author of more than 50 papers in refereed journals.

Paul Leiderer received his diploma
and Ph. D. in 1973 at the Technical
University of Munich (TUM). After
a research stay at Cornell in Ithaca,
New York, and habilitation at TUM
he was appointed professor in Mu-
nich in 1981 and at the University of
Mainz in 1982 . Since 1988 he has
been a full professor for experimen-

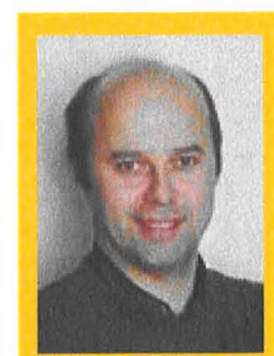

Johannes Boneberg received his diploma at the Ludwig-Maximilians University in Munich in 1988. In 1989 he joined the University of Konstanz, where he obtained his Ph. D. in 1993 and his habilitation in 1998. After a two year trip to the optical industry in Switzerland he returned to the physics department at the University of Konstanz, where he was appointed associate professor in 2006. His research interests are nanostructures and their interaction with light. He is an author or co-author of more than 80 papers in refereed journals. 


\section{References}

[1] B. C. Stuart, M. D. Feit, S. Herman, A. M. Rubenchik, B. W. Shore, and M. D. Perry, J. Opt. Soc. Am. B 13, 459 (1996).

[2] D. Bäuerle, Laser Processing and Chemistry (Springer, Berlin, 2000)

[3] S. Georgiou and A. Koubenakis, Chem. Rev. 103, 349 (2003).

[4] L. V. Zhigilei and E. Leveugle, Chem. Rev. 103, 321 (2003).

[5] P. Lorazo, L. J. Lewis, and M. Meunier, Phys. Rev. Lett. 91 , 225502 (2003).

[6] P. Lorazo, L. J. Lewis, and M. Meunier, Phys. Rev. B 73, $134108(2006)$.

[7] T. E. Glover, G. D. Ackerman, R. W. Lee, and D. A. Young, Appl. Phys. B 78, 995 (2004).

[8] B. J. Garrison, T. E. Itina, and L. V. Zhigilei, Phys. Rev. E 68, 041501 (2003).

[9] A. Miotello and R. Kelly, Appl. Phys. A 69, 67 (1999)

[10] F. Mafune, J. Kohno, Y. Takeda, and T. Kondow, J. Phys. Chem. B 106, 7575 (2002).

[11] A. Cavalleri, K. Sokolowski-Tinten, J. Bialkowski, M. Schreiner, and D. von der Linde, J. Appl. Phys. 85, 3301 (1999)

[12|J. Bonse, S. Baudach, J. Krüger, W. Kautek, and M. Lenzner, Appl. Phys. A 74, 19 (2002).

[13] C. Ropers, D. R. Solli, C. P. Schulz, C. Lienau, and T. ElSaesser, Phys. Rev. Lett. 98, 043907 (2007)

[14] D. von der Linde and K. Sokolowski-Tinten, Appl. Surf. Sci. 154-155, I (2000).

[15] D. Perez and L. J. Lewis, Phys. Rev. Lett. 89, 255504-1 (2002)

[16] B. J. Siwick, J. R. Dwyer, R. E. Jordan, and R. J. D. Miller, Science 302, 1382 (2003).

[17] A. Arbouet, C. Voisin, D. Christofilos, P. Langot, N. D. Fatti, F. Vallée, J. Lermé, G. Celep, E. Cottancin, M. Gaudry, M. Pellarin, M. Broyer, M. Maillard, M.P. Pileni, and M. Treguer, Phys. Rev. Lett. 90(17), 177401 (2003).

[18] J. Hodak, I. Martini, and G. V. Hartland, Chem. Phys. Lett. 284, 135 (1998)

[19] K. Sokolowski-Tinten, I. Bialkowski, and D. von der Linde, Phys. Rev. B 51, 14186 (1995).

[20] C. V. Shank, R. Yen, and C. Hirlimann, Phys. Rev. Lett. 50, 454 (1983)

[21] K. Sokolowski-Tinten, C. Blome, C. Dietrich, A. Tarasevitch, M. H. von Hoegen, D. von der Linde, A. Cavalleri, J. Squier, and M. Kammler, Phys. Rev. Lett. 87, 225701 (2001).

[22] A. Rousse, C. Rischel, S. Fourmaux, I. Uschmann, S. Sebban, G. Grillon, P. Balcou, E. Förster, J. Geindre, P. Audebert, J. Gauthier, and D. Hulin, Nature 410, 65 (2001).

[23] A. M. Lindenberg, J. Larsson, K. Sokolowski-Tinten, K. J. Gaffney, C. Blome, O. Synnergren, J. Sheppard, C. Caleman, A. G. MacPhee, D. Weinstein, D. P. Lowney, T. K. Allison, T. Mathews, R. W. Falcone, A. L. CavaIieri, D. M. Fritz, S. H. Lee, P. H. Bucksbaum, D. A. Reis, J. Rudati, P. H. Fuoss, C. C. Kao, D. P. Siddons, R. Pahl, J. Als-Nielsen, S. Duesterer, R. Ischebeck, H. Schlarb, H. Schulte-Schrepping, T. Tschentscher, J. Schneider, D. von der Linde, O. Hignette, F. Sette, H. N. Chapman, R. W. Lee, T.N. Hansen, S. Techert, J.S. Wark,
M. Bergh, G. Huldt, D. van der Spoe, N. Timneanu, J. Hajdu, R. A. Akre, E. Bong, P. Krejcik, J. Arthur, S. Brennan, K. Luening, and J. B. Hastings, Science 308, 392 (2005).

[24] E. S. Zijlstra, J. Walkenhorst, and M. E. Garcia, Phys. Rev. Lett. 101, 135701 (2008).

[25] M. Miller, Atom Probe Tomography (Kluwer Academic, New York, 2000).

[26] D. Blavelte, A. Bostel, J. M. Sarrau, B. Deconihout, and A. Menand, Nature 363, 432 (1993).

[27] C. S. Chang, W. B. Su, and T. T. Tsong, Phys. Rev. Lett. 72, 547 (1994).

[28] B. Gault, F. Vurpillot, A. Bostel, A. Menand, and B. Deconihout, Appl. Phys. Lett. 86, 094101 (2005)

[29] A. Vella, M. Gilbert, A. Hideur, F. Vurpillot, and B. Deconihout, Appl. Phys. Lett. 89, 251903 (2006).

[30] A. Vella, B. Deconihout, L. Marrucci, and E. Santamato, Phys. Rev. Lett. 99, 046103 (2007).

[31] H. Dachraoui and W. Husinsky, Appl. Phys. Lett. 89, $104102(2006)$.

[32] M. Merschdorf, C. Kennerknecht, K. Willig, and W. Pfeiffer, New J. Phys. 4, 95.1 (2002).

[33] T. Fennel, T. Döppner, J. Passig, C. Schaal, J. Tiggesbäumker, and K.H. Meiwes-Broer, Phys. Rev. Lett. 98, 143401 (2007)

[34] M. I. Stockman, S. V. Faleev, and D. J. Bergman, Phys. Rev. Lett. 88, 067402 (2002).

[35] M. Aeschlimann, M. Bauer, D. Bayer, T. Brixner, F. Javier Garcia de Abajo, W. Pfeiffer, M. Rohmer, C. Spindler, and F. Steeb, Nature 446, 301 (2007).

[36] M.I. Stockman, M. F. Kling, U. Kleineberg, and F. Krausz, Nature Photon. 1, 539 (2007).

[37] S. Nolte, B. N. Chichkov, H. Welling, Y. Shani, K. Lieberman, and H. Terkel, Opt. Lett. 24, 914 (1999).

[38] F. Zenhausern, Y. Martin, and H. K. Wickramasinghe, Science 269, 1083 (1995)

[39] T. Kalkbrenner, M. Ramstein, J. Mlynek, and V. Sandoghdar, J. Microsc. 202, 72 (200I).

[40] T. Kalkbrenner, U.H. Kanson, A. Schädle, S. Burger, C. Henkel, and V. Sandoghdar, Phys. Rev. Lett. 95, 200801 (2005).

[41] J. Jersch and K. Dickmann, Appl. Phys. Lett. 68, 868 (1996)

[42] J. Boneberg, H.J. Münzer, M. Tresp, M. Ochmann, and P. Leiderer, Appl. Phys. A 67, 381 (1998).

[43] R. Huber, M. Koch, and J. Feldmann, Appl. Phys. Lett. 73, 2521 (1998).

[44] A. Kirsanov, A. Kiselev, A. Stepanova, and N. Polushkin, J. Appl. Phys. 94, 6822 (2003).

[45] A. Chimmalgi, C. Grigoropoulos, and K. Komvopoulos, J. Appl. Phys. 97, 104319 (2005).

[46] G. Mie, Ann. Phys 4, 377 (1908).

[47] P. B. Johnson and R. W. Christy, Phys. Rev. B 6, 4370 (1972).

[48] D. Lynch and W. Hunter, Handbook of Optical Constants of Solids (Academic Press, New York, 1985).

[49] U. Kreibig and M. Vollmer, Optical Properties of Metal Clusters (Springer, Berlin, 1995).

[50] M. Perner, P. Bost, U. Lemmer, G. von Plessen, J. Feldmann, U. Becker, M. Mennig, M. Schmitt, and H. Schmidt, Phys. Rev. Lett. 78, 2192 (1997). 
[51] J. Hodak, I. Martini, and G. V. Hartland, Chem. Phys. Lett. 284, 135 (1998)

[52] N. del Fatti, C. Voisin, F. Chevy, F. Vallée, and C. Flytzanis, J. Chem. Phys. 110, 11484 (1999).

[53] T. Klar, M. Perner, S. Grosse, G. von Plessen, W. Spirkl, and J. Feldmann, Phys. Rev. Lelt. 80, 4249 (1998).

[54] C. Sönnichsen, T. Franzl, T. Wilk, G. von Plessen, and J. Feldman, New J. Phys. 4, 93.1 (2002).

[55] R. Rosei and D. W. Lynch, Phys. Rev. B 5, 3883 (1972).

[56] A. Taflove, Computational Electrodynamics: The FiniteDifference Time-Domain Method (Arrech House, Boston, MA, 1995).

[57] B. T. Draine and P. J. Flatau, J. Opt. Soc. Am. A 11, 1491 (1994).

[58] K. L. Shuford, M. A. Ratner, and G. Schatz, J. Chem. Phys. 123, 713 (2005).

[59] R. C. Jin, Y. C. Cao, E. C. Hao, G. S. Metraux, G. C. Schatz, and C. A. Mirkin, Nature 425, 487 (2003).

[60] C. L. Haynes, A. D. McFarland, L. Zhao, G.C. Schatz, R. P. Van Duyne, L. Gunnarsson, J. Prikulis, B. Kasemo, and M. Käll, J. Phys. Chem. B 107, 7337 (2003).

[61] J. Boneberg, J. König-Birk, H. J. Münzer, P. Leiderer, K. Shuford, and G. C. Schatz, Appl. Phys. A 89, 299 (2007).

[62] S. Link and M. A. El-Sayed, J. Phys. Chem. B 103, 4212 (1999).

[63] J. Kimling, M. Maier, B. Okenve, V. Kotaidis, H. Ballot, and A. Plech, J. Phys. Chem. B 110(32), 15700 (2006).

[64] R. Denk, K. Piglmayer, and D. Bäuerle, Appl. Phys. A 74, 825 (2002)

[65] D. Brodoceanu, L. Landström, and D. Bäuerle, Appl. Phys. A 86, $313(2007)$

[66] E. Prodan, C. Radloff, N. J. Halas, and P. Nordlander, Science 302, 419 (2003).

[67] P. Nordlander, C. Oubre, E. Prodan, K. Li, and M. Stockman, Nano Lett. 4(5), 899 (2004).

[68] M. Pelton, J. Aizpurua, and G. Bryant, Laser Photon. Rev. 2, $136(2008)$

[69] K. Ueno, S. Juodkazis, V. Mizeikis, D. Ohnishi, K. Sasaki, and H. Misawa, Opt. Express 15, 16527 (2007).

[70] P. K. Jain, W. Huang, and M. A. El-Sayed, Nano Lett. 7, 2080 (2007).

[71] J. Merlein, M. Kahl, A. Zuschlag, A. Sell, A. Halm, J. Boneberg, P. Leiderer, A. Leitenstorfer, and R. Bratschitsch, Nature Photon. 2, 230 (2008)

[72] J. N. Farahani, D. W. Pohl, H. J. Eisler, and B. Hecht, Phys Rev. Lett. 95, 017402 (2005)

[73] M. Kacmpfe, T. Rainer, K.J. Berg, G. Seifert, and H. Graener, Appl. Phys. Lett. 74, 1200 (1999).

[74] M. Kaempfe, H. Graener, A. Kiesow, and A. Heilmann, Appl. Phys. Lett. 79, 1876 (2001).

[75] A. Podlipensky, A. Abdolvand, G. Seifert, and H. Graener, Appl. Phys. A 80, 1647 (2005)

[76] C. J. Murphy, T. K. Sau, A. M. Gole, C. J. Orendorff, J. Gao, L. Gou, S. E. Hunyadi, and T. Li, J. Phys. Chem. B 109 $13857(2005)$

[77] C. Sönnichsen, B. M. Reinhard, J. Liphardt, and A. P. Alivisatos, Nature Biotechnol, 23, 741 (2005).

[78] J. Schmitt, P. Mächtle, D. Eck, H. Möhwald, and C. A. Helm, Langmuir 15, 3256 (1999).

[79] M. Perner, S. Grésillon, J. März, G. von Plessen, J. Feldmann, J. Porstendorfer, K. J. Berg, and G. Berg, Phys. Rev. Lett. 85, 792 (2000).
[80] G. V. Hartland, M. Hu, and J. E. Sader, J. Phys. Chem. B 107, $7472(2003)$.

[81] S. Link and M. A. El-Sayed, Int. Rev. Phys. Chem. 19, 409 (2000)

[82] G. Seifert, M. Kaempfe, K. J. Berg, and H. Graener, Appl. Phys. B 71, 795 (2000).

[83] G. Seifert, M. Kaempfe, K. J. Berg, and H. Graener, Appl Phys. B 73, 355 (2001).

[84] H. Graener, A. Podlipensky, G. Seifert, M. Leitner, and B. Sepiol, Quantum Electronics and Laser Science Conference 2007 (QELS '07), 1-2 May 2007.

[85] T. Döppner, T. Fennel, T. Diederich, J. Tiggesbäumker, and K. H. Mciwes-Brocr, Phys. Rev. Lett. 94, 013401 (2005).

[86] C. Rischel, A. Rousse, I. Uschmann, P. A. Albouy, J.P. Geindre, P. Audebert, J.C. Gauthier, E. Förster, J. L. Martin, and A. Antonetti, Nature 390, 490 (1997).

[87] K. Sokolowski-Tinten, C. Blome, J. Blums, A. Cavalleri, C. Dietrich, A. Tarasevitch, I. Uschmann, E. Förster, M. H. von Hoegen, and D. von der Linde, Nature 422, 287 (2003)

[88] F. Schotte, S. Techert, P. A. Anfinrud, V. Srajer, K. Moffat, and M. Wulff, in: Third-Generation Hard X-ray Synchrotron Radiation Sources, edited by D. Mills (John Wiley, Berlin, 2002).

[89] M. Wulff, F. Schotte, G. Naylor, D. Bourgeois, K. Moffat, and G. Mourou, Nucl. Instrum. Methods Phys. Res. A 398 69 (1997).

[90] L. Eybert, M. Wulff, W. Reichenbach, A. Plech, F. Schotte, E. Gagliardini, L. Zhang, O. Hignette, A. Rommeveaux, and A. Freund, Proc. SPIE 4782, 246 (2002).

[91] A. Plech, V. Kotaidis, K. Istomin, and M. Wulff, J. Synchrotron Radiat. 90, 022905 (2007).

[92] J. Turkevich, P. C. Stevenson, and J. Hillier, Discuss. Faraday Soc. 11, 55 (1951).

[93] J. Turkevich, P.C. Stevenson, and J. Hillier, Discuss. Faraday Soc. 57, 670 (1953).

[94] G. Frens, Nature Phys. Sci. 20, 241 (1973).

[95] U. C. Fischer and H. P. Zingsheim, J. Vac. Sci. Technol. 19, $88 \mathrm{I}(1981)$

[96] F. Burmeister, C. Schäfle, T. W. Matthes, M. Böhmisch, J. Boneberg, and P. Leiderer, Langmuir 13, 2983 (1997).

[97] F. Burmeister, C. Schaefle, B. Keilhofer, C. Bechinger, J. Boneberg, and P. Leiderer, Chem. Eng. Technol. 21, 761 (1998).

[98] A. D. McFarland, M. A. Young, J.A. Dieringer, and R. P. V. Duyne, J. Phys. Chem. B 109, 11279 (2005).

[99] O. M. Wilson, X. Hu, D. G. Cahill, and P. V. Braun, Phys Rev, B 66, 224301 (2002)

[100] G. V. Hartland, J. Chem. Phys. 116, 8048 (2002).

[101] A. Plech, M. Wulff, S. Kuerbitz, K. J. Berg, G. Berg, H. Graener, S. Grésillon, M. Kaempfe, J. Feldmann, and G. von Plessen, Europhys. Lett. 61, 762 (2003).

[102] Z. Ge, D. G. Cahill, and P. V. Braun, J. Phys. Chem. B 108 , 18870 (2004).

[103] A. Plech, V. Kotaidis, S. Grésillon, C. Dahmen, and G. von Plessen, Phys. Rev. B 70, 195423 (2004).

[104] V. Kotaidis, C. Dahmen, G. von Plessen, F. Springer, and A. Plech, J. Chem. Phys. 124, 184702 (2006).

[105] O. Ekici, R. K. Harrison, N. J. Durr, D. S. Eversole. M. Lee, and A. Ben-Yakar, J. Phys. D: Appl. Phys. 41, 185501 (2008) 
[106] A. Plech, A. Siems, V. Kotaidis, and A. Menzel, Proc. SPIE 6879, 68790H (2008).

[107] Y. S. Touloukian, R. K. Kirby, R. E. Taylor, and P. D. Desai, in: Thermal Expansion - Metallic Elements and Alloys (IFI Plenum, New York, 1975).

[108] C. Y. Ruan, Y. Murooka, R. K. Raman, and R. A. Murdick, Nano Lett. 7, 1290 (2007).

[109] J. Boneberg, J. Bischof, and P. Leiderer, Opt. Commun. $174,145(2000)$

[110] A. H. Zewail, Annu. Rev. Phys. Chem. 57, 65 (2006).

[111] H. Thee, V. A. Lobastov, U. M. Gomez, B. M. Goodson, R. Srinivasan, C. Y. Ruan, and A. H. 7cwail, Science 291, 458 (2001).

[112] S. Nie, X. Wang, H. Park, R. Clinite, and J. Can, Phys. Rev. Lett. 96, 025901 (2006).

[113] A. Plech, S. Grésillon, G. von Plessen, K. Scheidt, and G. Naylor, Chem. Phys. 299, 183 (2004).

[114] A. Plech, V. Kotaidis, M. Lorenc, and J. Boneberg, Nature Phys. 2, 44 (2006).

[115] A. Guinier and G. Fournet, Small-angle Scattering of Xrays (John Wiley, New York, 1955).

[116] V. Kotaidis and A. Plech, Appl. Phys. Lett. 87, 213102 (2005).

[117] P. Leiderer, C. Bartels, J. König-Birk, M. Mosbacher, and J. Boneberg, Appl. Phys. Lett. 85, 5370 (2004).

[118] M. Mosbacher, H.J. Münzer, J. Zimmermann, J. Solis, J. Boneberg, and P. Leiderer, Appl. Phys. A 72, 41 (2001).

[119] H. J. Münzer, M. Mosbacher, M. Bertsch, J. Zimmermann, P. Leiderer, and J. Boneberg, J. Microsc. 202, 129 (2001).
[120] N. Nedyalkov, T. Sakai, T. Miyanishi, and M. Obara, J. Phys. D 39, 5037 (2006).

[121] D. Eversole, B. Luk'yanchuk, and A. Ben-Yakar, Appl. Phys. A 89, 283 (2007)

[122] N. Nedyalkov, P. A. Atanasov, and M. Obara, Nanotechnology $18,305703(2007)$.

[123] N. N. Nedyalkov, T. Miyanishi, and M. Obara, Appl. Surf. Sci. 253, 6558 (2007).

[124] H. Takada and M. Obara, Jpn. J. Appl. Phys. 44, 9773 (2005).

[125] O.W. O, T. Ikawa, M. Hasegawa, M. Tsuchimori, and Y. Kawata, Appl. Phys. Lett. 79, 1366 (2001).

[126] T. Ikawa, T. Mitsuoka, M. Hasegawa, M. T. O. Watanabe, and Y. Kawata, Phys. Rev. B 64, 195408 (2001).

[127] S. Theppakuttai and S. Chen, Appl. Phys. Lett. 83, 758 (2003).

[128] B. S. Luk'yanchuk, N. Arnold, S. M. Huang, Z. B. Wang, and M. H. Hong, Appl. Phys. A 77, 209 (2003).

[129] T. Sakai, N. Nedyalkov, and M. Obara, J. Phys. D: Appl. Phys. 40, 7485 (2007).

[130] A. Plech, T. Salditt, C. Münster, and J. Peisl, J. Colloid Interface Sci. 223, 74 (2000).

[131] K. Ueno, V. Mizeikis, S. Juodkazis, K. Sasaki, and H. Misawa, Opt. Lett. 30, 2158 (2005).

[132] W. Huang, W. Qian, and M. A. El-Sayed, J. Chem. Phys. 98, 114301 (2005).

[133] W. Huang, W. Qian, and M. A. El-Sayed, J. Am. Chem. Soc. 128, 13330 (2006). 